Journal of Advanced Research in Fluid Mechanics and Thermal Sciences

\title{
Characteristics of Spray Angle and Discharge Coefficient of Pressure-Swirl Atomizer
}

\author{
Zulkifli Abdul Ghaffar ${ }^{1}$, Salmiah Kasolang ${ }^{1,}{ }^{*}$, Ahmad Hussein Abdul Hamid ${ }^{1}$ \\ 1 Faculty of Mechanical Engineering, Universiti Teknologi MARA (UiTM), 40450 Shah Alam, Selangor, Malaysia
}

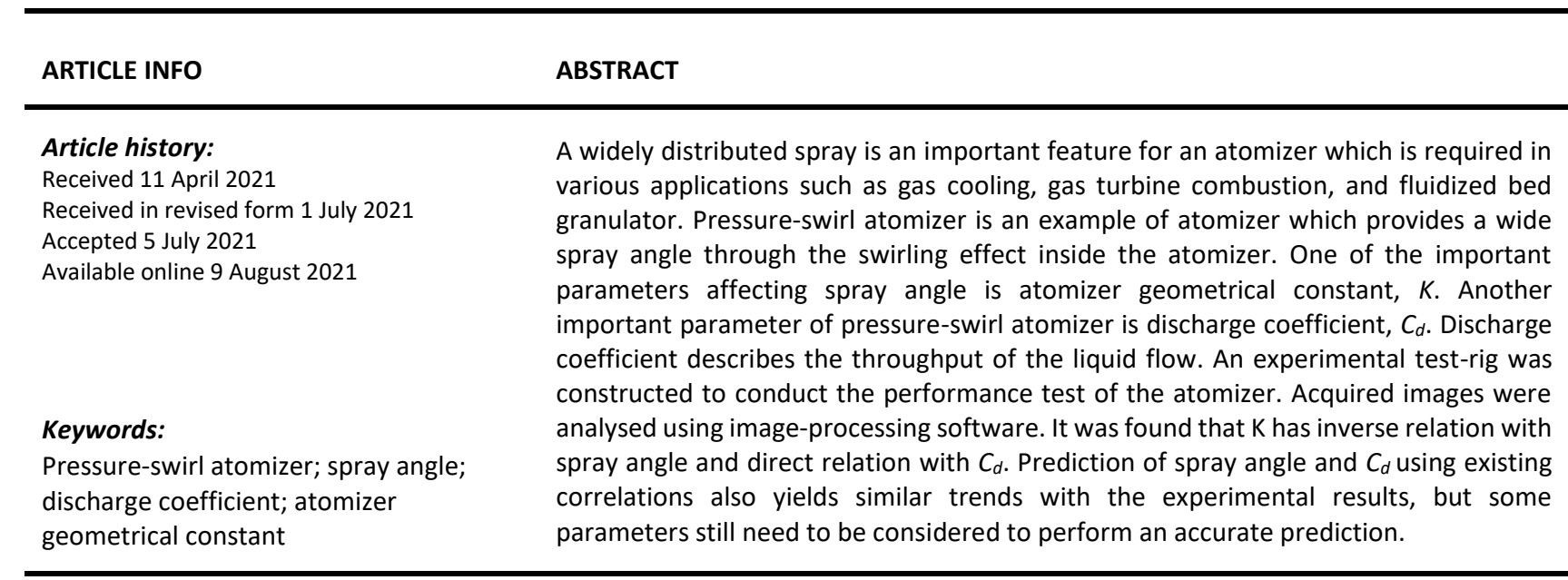

\section{Introduction}

Pressure-swirl atomizer is a widely applied atomizer for various industries. It is an example of atomizer which provides a wide spray angle induced by the swirling effect inside the atomizer. The schematic of pressure-swirl atomizer is shown in Figure 1. A wide spray angle is an important feature requires by various applications. For gas cooling applications, wider spray pattern of pressure-swirl atomizer combined with the slower velocity field will tend to allow for a more even mixing in the intake gases compared to the impinging jet atomizer [1]. In engine combustion [2-3], increase in spray angle increases the extent surrounding air exposure, leading to improved atomization, better fuelair mixing, and better dispersion of the fuel drops throughout the combustion volume [4]. A wider spray angle also requires in fluidized bed granulator which affects the area of wetted bed [5]. Spray angle is defined as the angle of the spray profile. Schematic of spray angle is shown in Figure 2.

\footnotetext{
* Corresponding author.

E-mail address: salmiahk@uitm.edu.my

https://doi.org/10.37934/arfmts.85.2.107114
} 


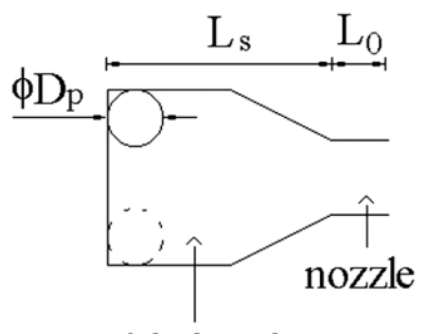

swirl chamber

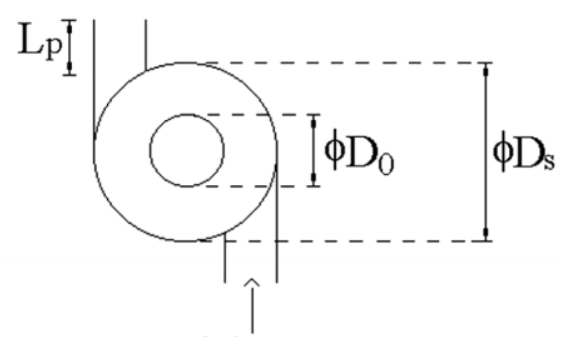

tangential passages

Fig. 1. Schematic of pressure-swirl atomizer [2]

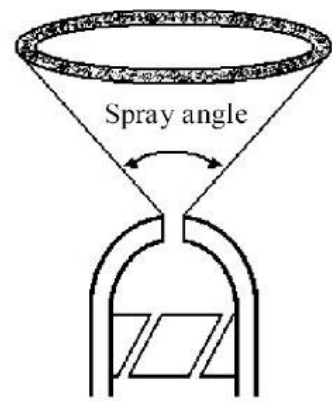

Fig. 2. Schematic of spray angle [6]

Research on pressure-swirl atomizer was conducted to study the effect of various parameters on the resultant spray angle. One of the important parameters affecting spray angle is called atomizer geometrical constant, $K$ and defined as:

$K=\frac{A_{p}}{D_{o} D_{s}}$

where $A_{p}$ is the tangential inlet port area, $D_{o}$ is the orifice diameter, and $D_{s}$ is the swirl chamber diameter.

Rizk and Lefebvre [7] investigated the influence of $K$ on spray angle which covers the range of 0.1 $\leq K \leq 0.4$ and observed the inverse relation of spray angle with $K$. Rizk and Lefebvre [7] also proposed empirical correlation which is in agreement with the findings of Dombrowski and Hasson [8]. Liao et al., [9] has also observed the same trends with Rizk and Lefebvre [7] upon investigating the effect of $K$ on spray angle. Rizk and Lefebvre [7] proposed correlation of spray angle, $2 \theta$ is:

$2 \theta=6 K^{-0.15}\left(\frac{\Delta P D_{o}^{2} \rho_{L}}{\mu_{L}^{2}}\right)^{0.11}$

where $K$ is atomizer geometrical constant, $\Delta P$ is the pressure drop, $D_{0}$ is the orifice diameter, $\rho_{L}$ is the liquid density, and $\mu_{\mathrm{L}}$ is the liquid dynamic viscosity.

Atomizer geometrical constant, $K$ has also been an important parameter in investigating discharge coefficient, $C_{d}[10]$. Discharge coefficient allows prediction of the actual liquid flow rate for given operating conditions [11]. It is defined as the ratio of actual mass flowrate to the theoretical mass flowrate or mathematically, 


$$
C_{d}=\frac{\text { Actual mass flowrate }}{\text { Theoretical mass flowrate }}=\frac{\dot{m}_{L}}{A_{o} \sqrt{2 \Delta P_{L} \rho_{L}}}
$$

where $\dot{\mathrm{m}}_{L}$ is the liquid mass flowrate, $\mathrm{kg} / \mathrm{s}, \mathrm{A}_{\circ}$ is the total discharge orifice area, $\Delta P$ is the pressure drop, and $\rho_{\mathrm{L}}$ is the liquid density.

Discharge coefficient was predicted by Rizk and Lefebvre [12] as a function of $K$ in a correlation:

$$
C_{d}=0.35 \cdot \sqrt{K} \cdot \sqrt[4]{\frac{1}{N}}
$$

where $K$ is the atomizer geometrical constant and $\mathrm{N}$ is the orifice-to-swirl chamber diameter ratio $\left(D_{0} / D_{s}\right)$.

The study on the effect of atomizer geometrical constant, $K$ on spray angle and discharge coefficient was previously conducted and empirical correlation was proposed. However, the proposed empirical correlation still requires verification of its suitability of any system. Hence, this present study aims to conduct experimental investigation on the effect of atomizer geometrical constant, $K$ on spray angle and discharge coefficient of pressure-swirl atomizers and compare the acquired results with the proposed empirical correlation to verify the empirical correlations.

\section{Methodology}

The investigated pressure-swirl atomizer has three tangential inlet slots and fabricated from acrylic rod. Post-machining was performed using abrasive paper and polishing liquid to ensure a smooth surface and good transparency. Transparency is crucial for accurate internal flow visualizations and characterizations. The dimensions of $D_{p}, D_{s}, D_{0}, L_{s}, L_{0}$, and $\alpha$ for the investigated atomizers are shown in Table 1.

Table 1

Dimensions of the tested atomizers

\begin{tabular}{lllllll}
\hline No. & $\begin{array}{l}D_{p} \\
(\mathrm{~mm})\end{array}$ & $\begin{array}{l}\mathrm{D}_{\mathrm{s}} \\
(\mathrm{mm})\end{array}$ & $\begin{array}{l}\mathrm{D}_{\circ} \\
(\mathrm{mm})\end{array}$ & $\begin{array}{l}\mathrm{L}_{\mathrm{s}} \\
(\mathrm{mm})\end{array}$ & $\begin{array}{l}\mathrm{L}_{o} \\
(\mathrm{~mm})\end{array}$ & $\alpha\left(^{\circ}\right)$ \\
\hline 1 & 3.00 & 20 & 3.00 & 85 & 15 & 66 \\
2 & 3.00 & 20 & 5.00 & 85 & 15 & 60 \\
3 & 3.00 & 20 & 7.00 & 85 & 15 & 53 \\
\hline
\end{tabular}

An experimental test-rig was manufactured as a platform for the atomizer performance test. Water was used as the working fluid considering its availability and ease of handling. A pulseless centrifugal pump was utilized for delivering water from the water supply tank to the atomizer through the waterline. The amount of water flowing out of the pump was controlled by a ball valve installed at the outlet. Measurement of water flow rates in the system was obtained from water flow transmitter. The flow of water was controlled by globe valve. Water strainer was installed prior to the water flow transmitter inlet to prevent unwanted debris passing through the meter and led to malfunctioned [13]. Water injection pressure was measured by digital pressure gauges. The injector was fixed in vertical downward position and produce water sprays into a water collection tank. A line diagram of the experimental test-rig is shown in Figure 3. High-speed shadowgraph technique was applied using high resolution camera for visualization of internal (air core) and external flow (spray angle) parameters. A flashlight was arranged with pointing towards the camera lens aperture. 
Measurement of spray angle was adapted from our previous research [14]. It was performed by converting the spray images to binary form to facilitate the visualizations of the spray boundary as shown in Figure 4. Angle measurement tool was utilized in determining the spray angle as depicted in Figure 5.

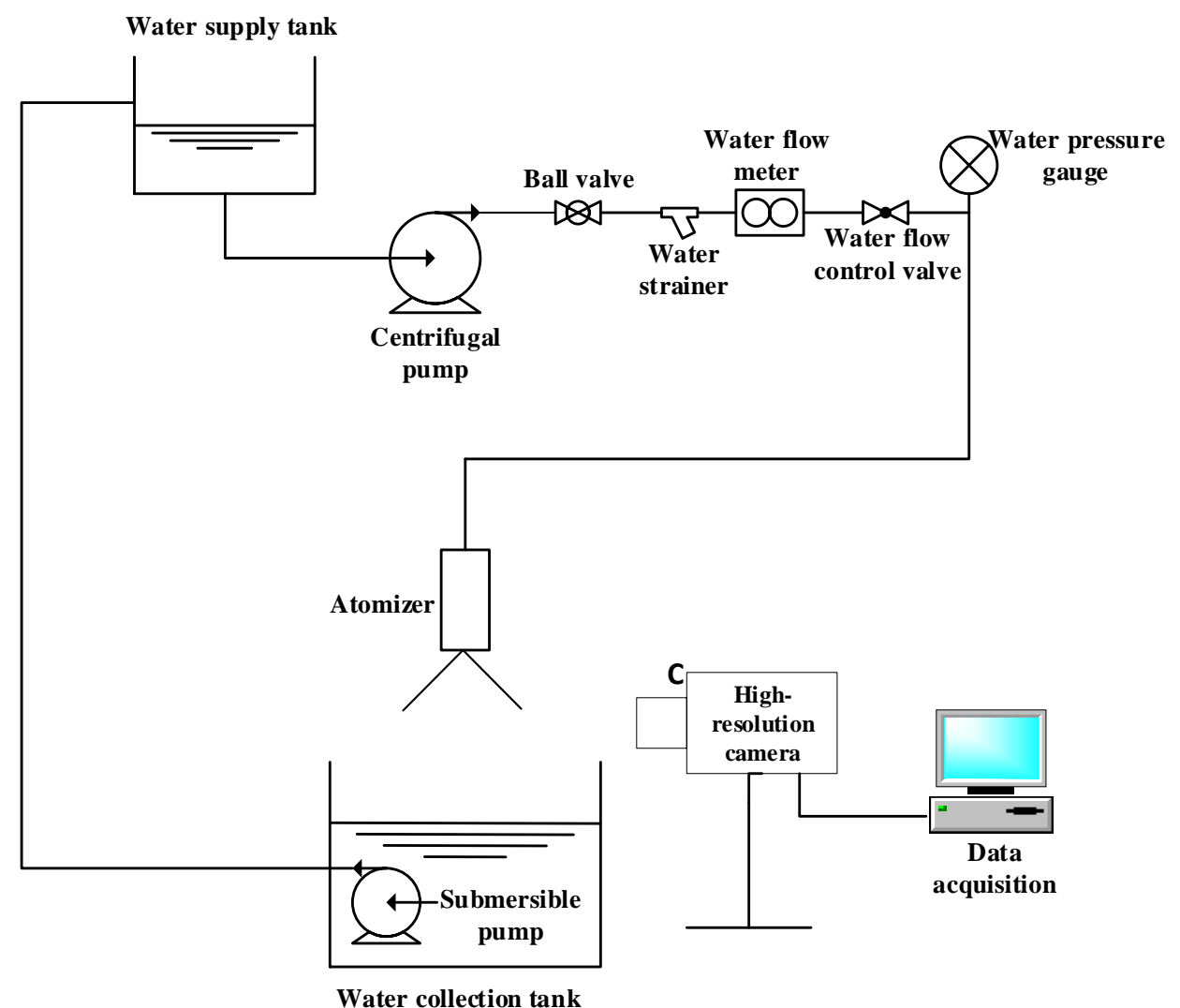

Fig. 3. Line diagram of the experimental test-rig

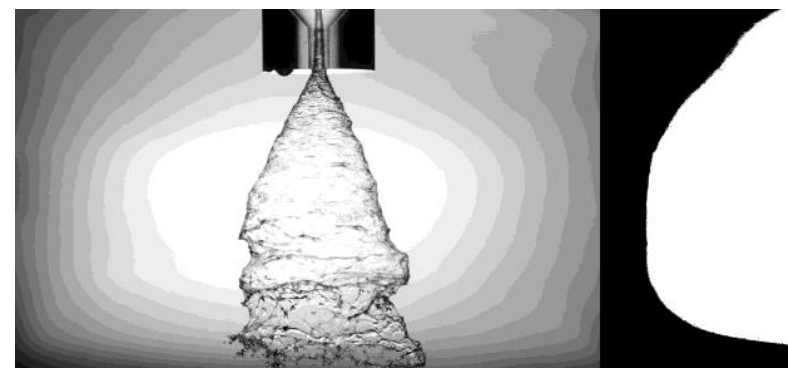

(a)

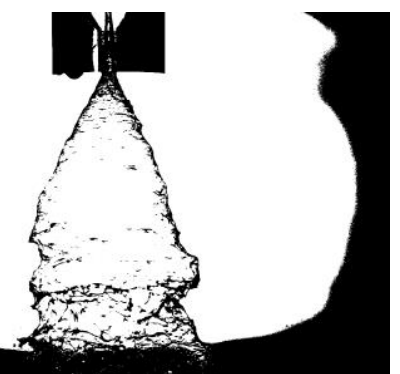

(b)

Fig. 4. (a) Original and (b) binary converted spray image

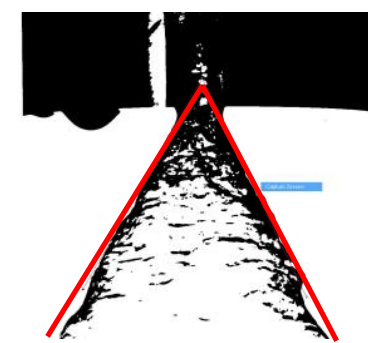

Fig. $\quad 5 . \quad$ Angle

measurement tool

(highlighted in red) 


\section{Results}

The results are presented in three sub-sections, which are: 3.1 Spray angle, 3.2 Discharge coefficient, and 3.3 Prediction of spray angle and discharge coefficient using existing correlations.

\subsection{Spray Angle}

The effect of $K$ on spray angle is presented in Figure 6 . It is clearly observed that the spray angle is inversely proportional to the atomizer geometrical constant, $K$. The experimental finding has almost similar trend to the research findings by Rizk and Lefebvre [7] and Liao et al., [9]. However, the present experiment observed narrower sprays than the previous research findings for the same atomizer geometrical constant. This could be attributed to the value of $\Delta \mathrm{P}$ in Rizk and Lefebvre [7] and Liao et al., [9] experiments were higher than the present research. Higher $\Delta \mathrm{P}$ promotes higher tendency for turbulence to occur with growth in the liquid sheet momentum and components of velocity [15]. Effect of $\Delta \mathrm{P}$ on spray angle has also been investigated by various researchers [6-15-16] which concluded that increase in $\Delta \mathrm{P}$ enlarge the spray angle.

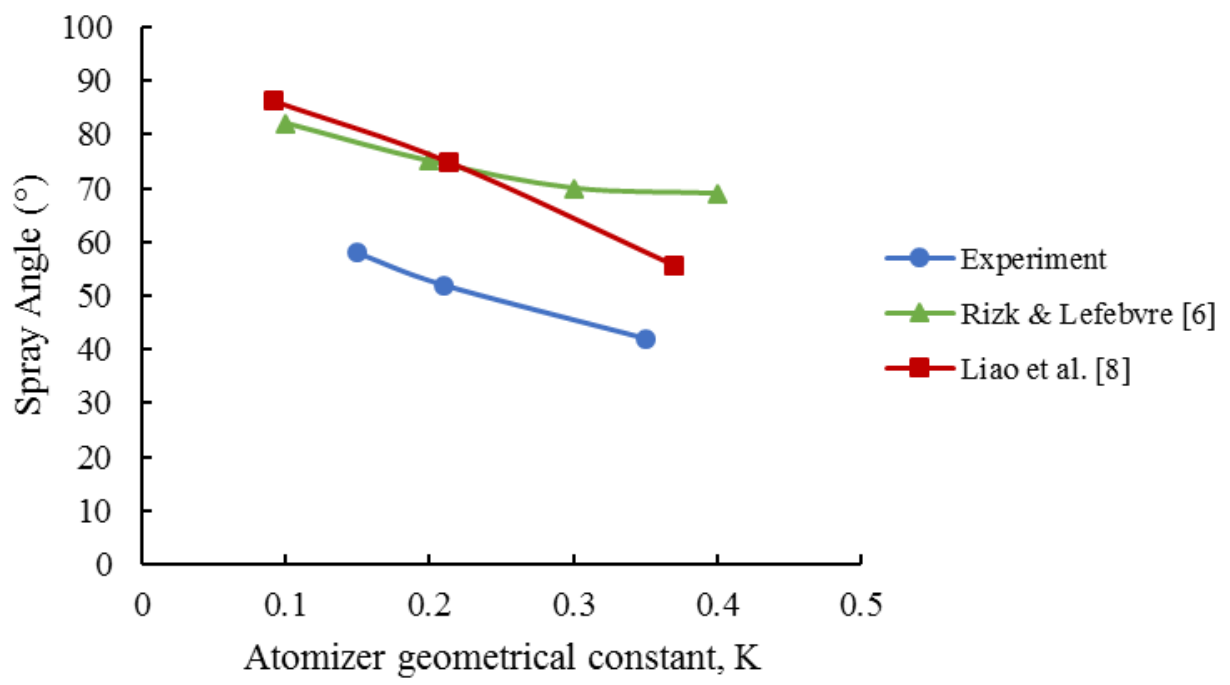

Fig. 6. Effect of $K$ on spray angle

\subsection{Discharge Coefficient}

The effect of $K$ on $C_{d}$ is presented in Figure 7. It is obviously spotted that $C_{d}$ is directly proportional to the atomizer constant $K$ with an upward trend. $C_{d}$ is a measure of atomizer efficiency at discharging liquid. It is also indicating the amount of friction exists within the system that has hindered an efficient liquid discharge. Value of $C_{d}$ equals to 1 characterize a perfect frictionless flow.

Comparison with previous research by Rizk and Lefebvre [12] and Liao et al., [9] showed that $C_{d}$ has a similar trend with present research by a direct proportional relation to $K$. The $C_{d}$ of Rizk and Lefebvre [12] and Liao et al., [9] has almost similar value along the increase of $K$ but the present research only depicted a similar value with Liao et al., [9] at $K=0.15$. Present research portrayed an abrupt increase in $C_{d}$ with $K$ as $K$ approaching 0.4 . The value of $C_{d}$ is 0.5 which is larger than both observed by Rizk and Lefebvre [12] and Liao et al., [9]. This indicates a more efficient liquid discharge from the present pressure-swirl atomizer. The probable reasoning of the phenomenon is the ratio of effective flow area, $A_{\text {eff }}$ to the discharge orifice, $D_{0}$ of the present research is larger. This allows more fluid discharging the orifice. The effective flow area, $A_{\text {eff }}$ can be defined as: 


$$
A_{\text {eff }}=\frac{\pi}{4}\left(D_{o}^{2}-D_{a}^{2}\right)
$$

where $D_{o}$ is the discharge orifice diameter and $D_{a}$ is the air core diameter.

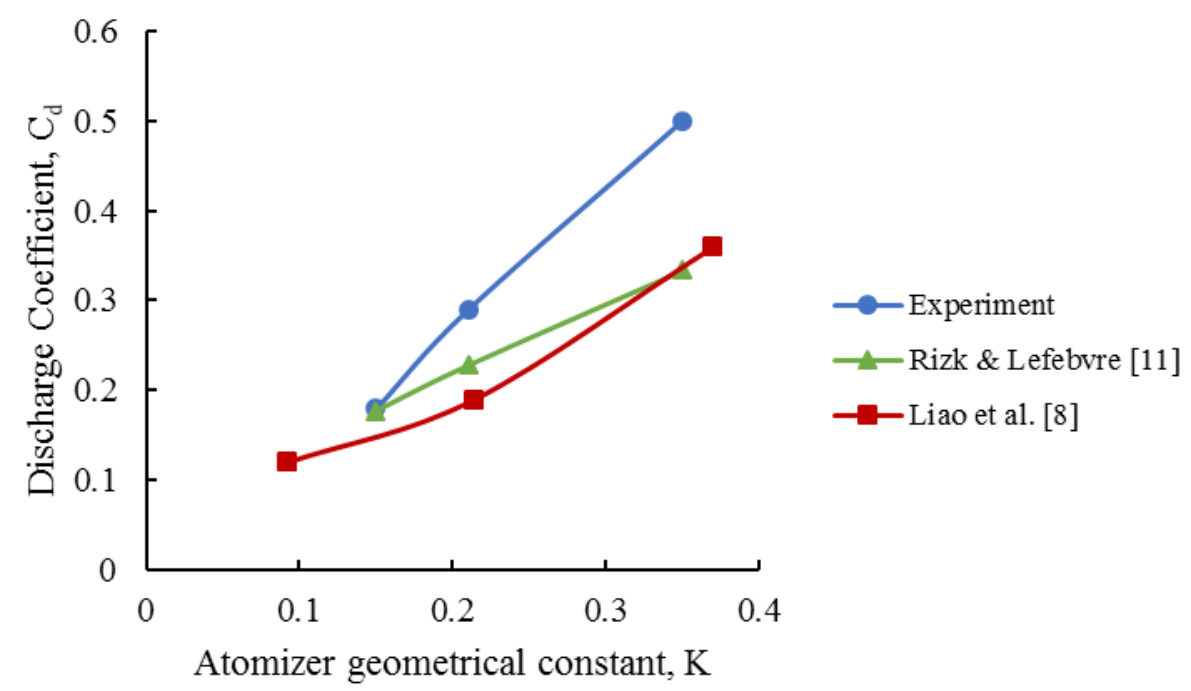

Fig. 7. Effect of $K$ on $C_{d}$

\subsection{Prediction of Spray Angle and Discharge Coefficient Using Existing Correlations}

Comparison of spray angle between the experimental results and calculated using existing empirical correlations proposed by Rizk and Lefebvre [7] is shown in Figure 8. It is observed that both has a similar trend; spray angle is inversely related to atomizer geometrical constant, $K$. However, the correlation does not accurately predict the spray angle. The empirical correlation seems to predict a larger spray angle value at every atomizer geometrical constant, $K$. This may suggest that the proposed empirical correlation is not universal and only suitable for a specific range of parameters, i.e., $\Delta \mathrm{P}$ and $\mathrm{D}_{\mathrm{o}}$.

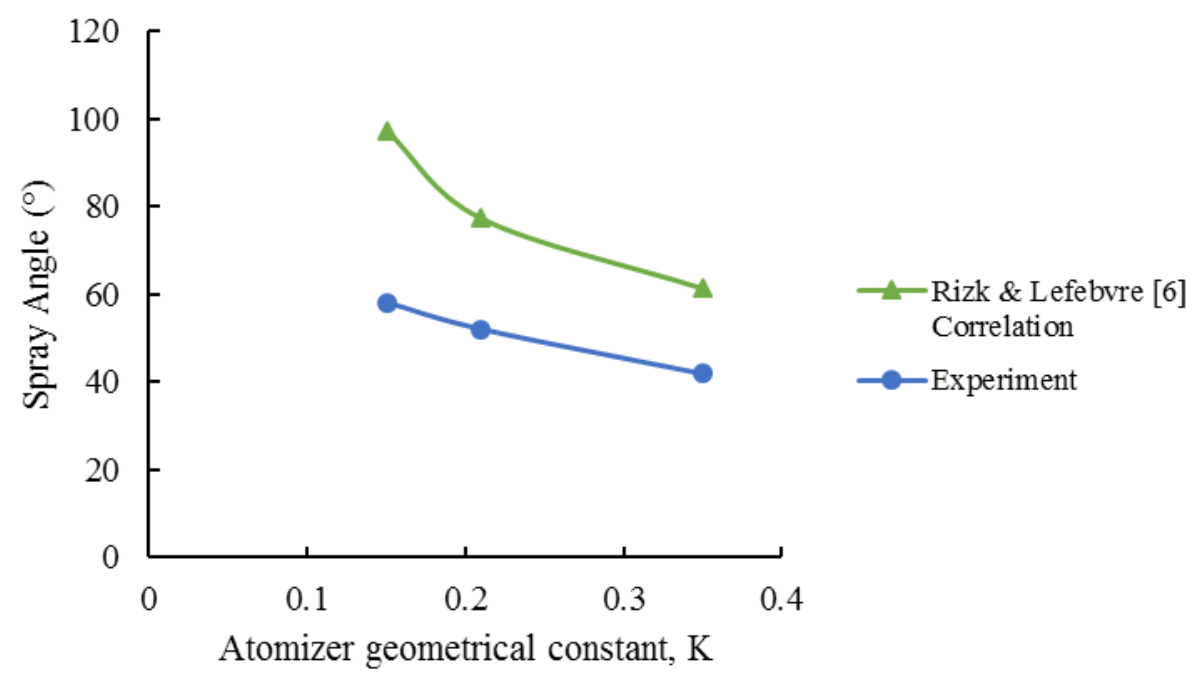

Fig. 8. Comparison of experiment and existing correlations on spray angle 
The experimental results and Rizk and Lefebvre [7] correlation of discharge coefficient, $C_{d}$ were compared as shown in Figure 9. It is observed that both results show a direct proportional trend but with different slope. Initially, both experimental and correlation show a similar result of $C_{d}$ but as atomizer geometrical constant increase, the experimental result depicted a higher $C_{d}$. This might indicate that the empirical correlation is lacking an important parameter known as effective flow area, $A_{\text {eff }}$ as discussed in previous sub-section.

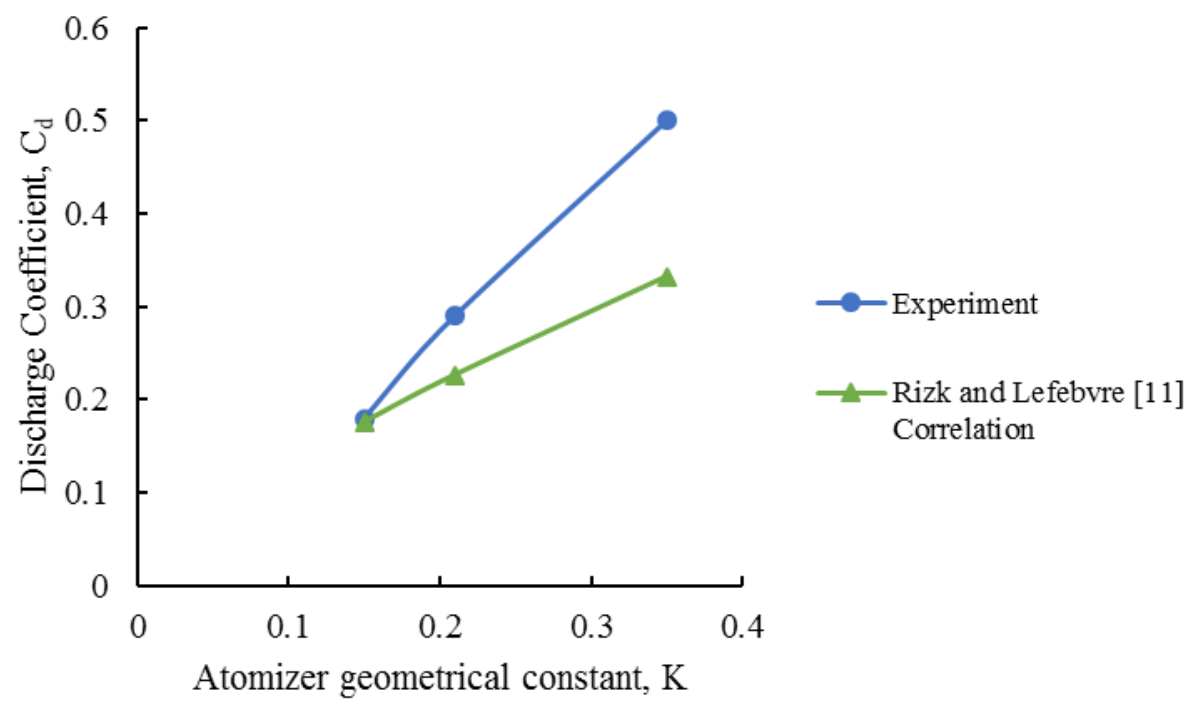

Fig. 9. Comparison of experiment and existing correlations on discharge coefficient

\section{Conclusions}

An experimental investigation was conducted to study the effect of atomizer geometrical constant on spray angle and discharge coefficient. The acquired results were compared with existing data from literature. The existing proposed empirical correlation was used to predict the spray angle and the obtained results were compared with experiments. It was found that the influence of atomizer geometrical constant, $K$ to the spray angle may also depends on pressure drop, $\Delta \mathrm{P}$. Although the value of $K$ in present research is the same with existing data from literature, the obtained spray angle is differs considering different value of pressure drop, $\Delta \mathrm{P}$. The investigation on effect of $K$ on $C_{d}$ revealed that a parameter also needs to be considered in predicting the $C_{d}$. This parameter known as effective flow area, $A_{\text {eff. }}$

\section{Acknowledgement}

This research was supported by the Ministry of Education Malaysia through Geran Penyelidikan Fundamental (FRGS), Reference number: 600-IRMI/FRGS 5/3 (395/2019). This acknowledgment is also extended to Research Management Center (RMC) of UiTM.

\section{References}

[1] Schick, Rudolf J., and Keith F. Knasiak. "Spray Characterization for Wet Compression Gas Cooling Applications." In Eighth International Conference on Liquid Atomization and Spray Systems, Pasadena, CA. 2000.

[2] Da Silva Couto, Heraldo, Pedro Teixeira Lacava, Demetrio Bastos-Netto, and Amilcar Porto Pimenta. "Experimental evaluation of a low pressure-swirl atomizer applied engineering design procedure." Journal of propulsion and power 25, no. 2 (2009): 358-364. https://doi.org/10.2514/1.37018 
[3] Jumadi, Rozita, Amir Khalid, Norrizam Jaat, Iqbal Shahridzuan Abdullah, Nofrizalidris Darlis, Bukhari Manshoor, Azahari Razali, Azwan Sapit, and Ridwan Saputra Nursal. "Analysis of Spray Characteristics and High Ambient Pressure in Gasoline Direct Injection using Computational Fluid Dynamics." CFD Letters 12, no. 5 (2020): 36-51. https://doi.org/10.37934/cfdl.12.5.3651

[4] Chen, S. K., A. H. Lefebvre, and J. Rollbuhler. "Factors influencing the effective spray cone angle of pressure-swirl atomizers." (1992): 97-103. https://doi.org/10.1115/1.2906313

[5] Juslin, Laura, Osmo Antikainen, Pasi Merkku, and Jouko Yliruusi. "Droplet size measurement: I. Effect of three independent variables on droplet size distribution and spray angle from a pneumatic nozzle." International journal of pharmaceutics 123, no. 2 (1995): 247-256. https://doi.org/10.1016/0378-5173(95)00081-S

[6] Hamid, Ahmad Hussein Abdul, and Rahim Atan. "Spray characteristics of jet-swirl nozzles for thrust chamber injector." Aerospace Science and Technology 13, no. 4-5 (2009): $192-196$. https://doi.org/10.1016/i.ast.2008.10.003

[7] Rizk, N. K., and A. H. Lefebvre. "Prediction of velocity coefficient and spray cone angle for simplex swirl atomizers." International Journal of Turbo and Jet Engines 4, no. 1-2 (1987): 65-74. https://doi.org/10.1515/TJJ.1987.4.1-2.65

[8] Dombrowski, Norman, and David Hasson. "The flow characteristics of swirl (centrifugal) spray pressure nozzles with low viscosity liquids." AIChE Journal 15, no. 4 (1969): 604-611. https://doi.org/10.1002/aic.690150424

[9] Liao, Y., A. T. Sakman, S. M. Jeng, M. A. Jog, and M. A. Benjamin. "A comprehensive model to predict simplex atomizer performance." (1999): 285-294. https://doi.org/10.1115/1.2817119

[10] Hong, Moongeun, Jaehyoung Jeon, and Soo Yong Lee. "Discharge coefficient of pressure-swirl atomizers with low nozzle opening coefficients." Journal of Propulsion and Power 28, no. 1 (2012): 213-218. https://doi.org/10.2514/1.B34168

[11] Hammad, Farid A., Kai Sun, Jan Jedelsky, and Tianyou Wang. "The Effect of Geometrical, Operational, Mixing Methods, and Rheological Parameters on Discharge Coefficients of Internal-Mixing Twin-Fluid Atomizers." Processes 8, no. 5 (2020): 563. https://doi.org/10.3390/pr8050563

[12] Rizk, N. K., and Arthur Henry Lefebvre. "Internal flow characteristics of simplex swirl atomizers." Journal of Propulsion and Power 1, no. 3 (1985): 193-199. https://doi.org/10.2514/3.22780

[13] Hedland, M. R. "Flow Transmitter Installations and Programming Instructions, vol." (2008).

[14] Ghaffar, Zulkifli Abdul, Salmiah Kasolang, and Ahmad Hussein Abdul Hamid. "Characteristics of swirl effervescent atomizer spray angle." In Applied Mechanics and Materials, vol. 607, pp. 108-111. Trans Tech Publications Ltd, 2014. https://doi.org/10.4028/www.scientific.net/AMM.607.108

[15] Dafsari, Reza Alidoost, Hyung Ju Lee, Jeongsik Han, Dong-Chang Park, and Jeekeun Lee. "Viscosity effect on the pressure swirl atomization of an alternative aviation fuel." Fuel 240 (2019): $179-191$. https://doi.org/10.1016/j.fuel.2018.11.132

[16] Zhang, Tao, Bo Dong, Xiaohong Chen, Zhonghua Qiu, Rui Jiang, and Weizhong Li. "Spray characteristics of pressureswirl nozzles at different nozzle diameters." Applied thermal engineering 121 (2017): 984-991. https://doi.org/10.1016/j.applthermaleng.2017.04.089 